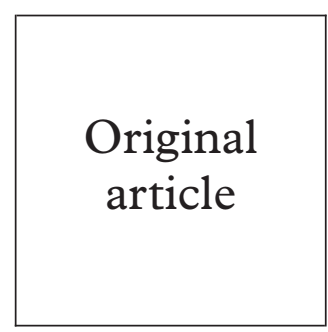

\author{
E Foley, V Harindra
}

\title{
Cervical cytology: are national guidelines adequate for women attending genitourinary medicine clinics?
}

Objectives: To study whether all women attending a genitourinary medicine (GUM) clinic warrant a cervical smear as part of a routine screen for infection, or whether this "at risk" population is adequately covered by the national screening programme.

Methods: A cervical smear and a screen for sexually transmitted infections (STI) were taken from 900 women attending a GUM clinic between May 1996 and April 1997.

Results: Of 812 smears available for analysis, 613 (75.5\%) were normal, $176(21.7 \%)$ were mildly abnormal, and $23(2.8 \%)$ were moderately or severely abnormal. In the absence of an STI there was a $14 \%(37 / 273)$ risk of having an abnormal cervical smear. In the presence of cervicitis the risk was $26 \%(22 / 84)$ and with genital warts the risk was $34 \%(75 / 215)$.

Conclusion: The national screening programme guidelines for cervical cytology should be followed in the GUM clinic. There is no benefit in performing extra smears outside the programme nor in adopting a policy of universal screening.

(Sex Transm Inf 1999;75:349-351)

Keywords: cervical cytology; sexually transmitted diseases; screening

\section{Introduction}

Cancer of the cervix is the eighth commonest cancer in women in the United Kingdom and the commonest cancer in women under 35 years of age. The ease with which precancer of the cervix can be detected allows a screening programme which fulfils Wilson and Junger's criteria. ${ }^{1}$ The coverage of the target population was inadequate until changes in remuneration for cervical screening in primary care were introduced. Currently in the United Kingdom more than $85 \%$ of women aged $20-64$ years are screened at intervals of 5 years.

The ability to identify and target a population at greater risk of developing invasive cervical cancer is attractive. Although the exact aetiology is not known, there are recognised risk factors associated with the development of cervical cancer and its precursor, cervical intraepithelial neoplasia (CIN). These include sexual intercourse before the age of 18 years, ${ }^{2}$ more than four sexual partners, and the presence of trichomonas infection. ${ }^{3}$ These risk factors are common in women attending a GUM clinic and it has been suggested that all should be offered a cervical smear as part of their routine screen for sexually transmitted infections (STI). ${ }^{45}$ The policy for cervical screening varies between genitourinary medicine (GUM) clinics. Many have a non-selective screening policy based on the presumption that these women have a low take up of the national programme. Others follow the national guidelines. ${ }^{6}$

The benefit of screening teenagers is uncertain. Teenagers do have cervical abnormalities which include high grade dysplasias but these do not seem to progress to carcinoma in situ (CIS) before the age of $21 .^{78}$ The national guidelines for screening puts the lower age limit at 20 years of age, as routine screening of teenagers is unlikely to have a significant impact on the mortality from cervical cancer, but may cause significant morbidity and psychological stress. A significant number of women attending a GUM clinic are under 20 years of age and, using a non-selective screening policy, this population would be included.

The purpose of this study was to determine whether all the women attending a GUM clinic warrant a cervical smear as part of a routine screen for infection, or whether this "at risk" population is adequately covered by the national screening programme.

\section{Patients and methods}

The protocol for cervical screening that operates in our clinic requires a cervical smear to be taken at the same time as an STI screen if the woman has not had a cervical smear in the previous year.

Clinical and sociodemographic information was collected for 900 consecutive cases where a cervical smear was obtained between May 1996 and April 1997. The patient's age, the indication for the smear, and the type of STI if present were recorded. Abnormal smear results were either repeated or the patient was referred for colposcopy. For the purpose of this study, only the most severe abnormality was recorded for each individual patient.

\section{CYTOLOGY}

Analysis of the cervical smears was performed and was classified according to the guidelines of the British Society for Clinical Cytology. ${ }^{9}$

\section{DATA ANALYSIS}

Selected sociodemographic, clinical, and cytological data were coded and stored in a 


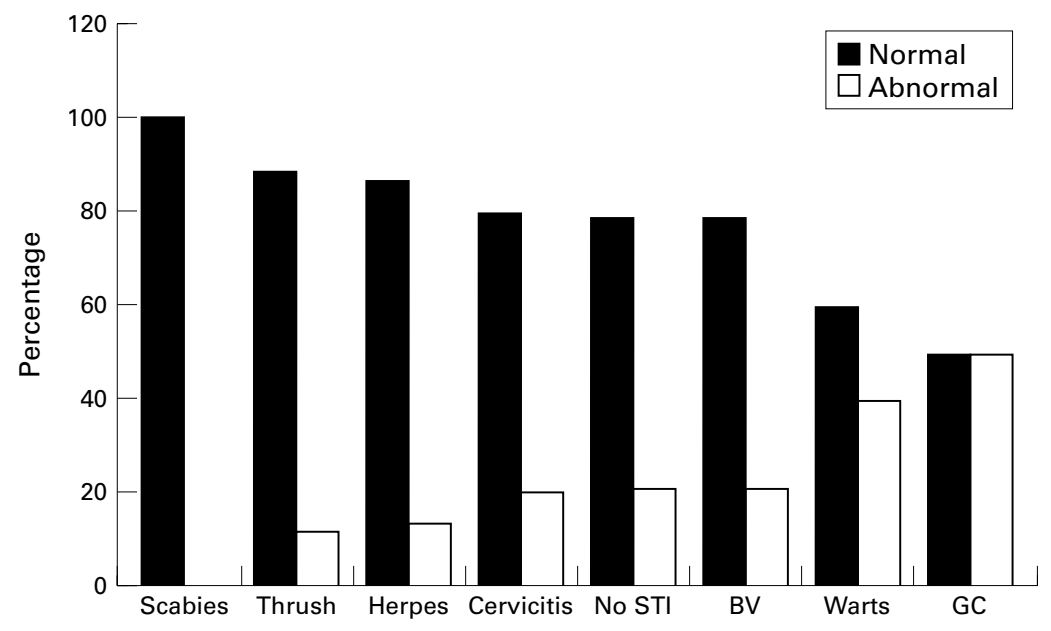

Figure 1 STI diagnosis and smear result.

computer database. Data were analysed using the SAS PC package.

\section{Results}

The age range of the study population was 14-61 years with a mean age of 27.2 years and a median age of 26 years.

For the whole sample, $59.8 \%$ of the cervical smears taken were for routine screening, 36\% for genital warts, and $4.2 \%$ for a previously abnormal smear result. In the 14-19 age group, $80 \%$ were performed because of the presence of genital warts and $20 \%$ for routine screening.

There was a non-diagnostic group of 88 $(9.7 \%)$ smears. Analysis was performed on the remaining 812 smears.

The majority of the smears taken (613/812, $75.5 \%)$ were reported as normal; $21.7 \%$ (176/ 812) were reported as borderline, mildly abnormal, or showed the presence of HPV; and $2.8 \%(23 / 812)$ were moderately or severely abnormal. For women aged between 14 and 19 years $60 \%(91 / 150)$ of the smears were reported to be normal; 37\% (55/150) were borderline, mildly abnormal, or showed the presence of HPV; and 3\% (4/150) showed either moderate or severe abnormalities.

Patients were assessed for the presence of an STI and the results are shown in figure 1 and table 1 . The cytology results were grouped as normal or abnormal. The majority of patients did not have a sexually transmitted infection (304/812). The most commonly found infection was genital warts $(236 / 812)$.

There was a $14 \%(37 / 273)$ risk of having an abnormal cervical smear in the absence of an STI. In the presence of cervicitis the risk was $26 \%(22 / 84)$ and when genital warts were

Table 1 STI diagnosis and cervical cytology result

\begin{tabular}{llllll}
\hline & Normal & $\%$ & Abnormal & $\%$ & Total \\
\hline No STI & 214 & 79 & 57 & 21 & 271 \\
Scabies & 2 & 100 & 0 & 0 & 2 \\
Thrush & 115 & 88.5 & 15 & 11.5 & 130 \\
Cervicitis & 75 & 79.8 & 19 & 20.2 & 94 \\
Bacterial vaginosis & 60 & 78.9 & 16 & 21.1 & 76 \\
Warts & 133 & 59.9 & 89 & 40.1 & 222 \\
Herpes & 13 & 86.7 & 2 & 13.3 & 15 \\
Gonococcus & 1 & 50 & 1 & 50 & 2 \\
Total & 613 & & 199 & & 812 \\
\hline
\end{tabular}

present the risk of a smear abnormality was $34 \%(75 / 215)($ linear $\times 2=28.7 \mathrm{p}<0.001)$.

As the most common indication for a cervical smear in the 14-19 year age group was the presence of genital warts this subgroup was analysed separately and compared with those women over 20 years of age with genital warts. There was no significant difference in the incidence of abnormal cytology between these two groups, 45/121 (37.2\%) compared with $28 / 94$ $(29.8 \%)$.

\section{Discussion}

In this population $24.5 \%$ of the cervical smears taken were abnormal. This is a higher proportion of abnormality than would be expected in the general population and would suggest that women attending a GUM clinic do indeed have a greater risk of a smear abnormality. Of the abnormal smear results, $88 \%$ showed only minor abnormalities and the majority of these would be expected to return to normal over time. ${ }^{10}$ Overall, only $2.8 \%$ of the smears taken were either moderately or severely abnormal. There were no cases of cervical cancer.

Increasingly, women attend a GUM clinic for reasons not related to STIs. Those without an STI at the time of screening are no more likely to have a cervical abnormality than women who have cervical cytology performed elsewhere $(14 \%$ versus $6-12 \%){ }^{1}$

The presence of cervicitis or genital warts doubles the likelihood of the smear being abnormal (26\% and $34 \%$ respectively). This result achieves statistical significance but, as the majority of these abnormalities are minor, it could be argued that any disease progression would be picked up through routine interval screening.

Of the women under the age of 20 years, $40 \%$ had an abnormal smear result. This implies that these "high risk" teenagers should be included in the screening programme. As the majority of these abnormalities were mildly dyskaryotic and most of the teenagers screened in our clinic had genital warts we infer that a policy of additional screening beyond the current nationally recommended age limits is unnecessary.

The national screening programme reaches $85 \%$ of the target population. ${ }^{6}$ Women attending GUM clinics have risk factors, which put them greater risk of developing cervical cancer, and may represent a proportion of those who fail to access health care elsewhere. GUM clinics are well placed to ensure that women are screened according to the national guidelines and the accredited colposcopy services they provide are complementary. This is particularly important when an STI is found. There is no benefit in performing extra smears outside the programme, and a policy of universal screening of women attending a GUM clinic is not justified.

1 Wilson JMG, Junger JJ. Principles and practice of screening for disease. WHO Public Health Paper. Geneva: WHO, 1968:34.

2 Parassini F, Hildesheim A, Ferraroni M, et al. Relative and attributable risk for cervical cancer: a comparative study in the United States and Italy. Int $\mathcal{F}$ Epidemiol 1990;19:53945 . 
3 Slattery M, Overall J, Abbott T, et al. Sexual activity, contraception, genital infections and cervical cancer: support fo a sexually transmitted disease hypothesis. Am I Epidemio 1989;130:248-58.

4 Campbell PJ, Hewitt SH, Kowalchuk PA, et al. Relationships of cervical cytologies to selected variables amongs women attending a sexually transmitted disease clinic. Int $f$ STD AIDS 1994;5:108-12.

5 Schwebke JR, Zajackowski ME. Effect of concurrent lower genital tract infections on cervical cancer screening. Genitourin Med 1997;73:383-6.

6 Duncan ID. Guidelines for clinical practice and programme management. Oxford: National Coordinating Network, mHS Cervical Screening Programme, 1993. MacGregor JE, Teper S. Uterine cervical cytology and young women. Lancet 1978;ii:1029-31.
8 Blomfield PI, Duncan ID. Cervical screening and teenage women. In: Bonnar J, ed. Recent advances in obstetrics and gynaecology. No 18. Edinburgh: Churchill Livingstone, 1994:155-60.

9 Evans DMD, Hudson EA, Brown CL, et al. Terminology in gynaecological cytopathology: report of the working party of the British Society for Clinical Cytology. 7 Clin Pathol 1986;39:933-44.

10 Holowaty P, Miller AB, Rohan T, et al. Natural history of dysplasia of the uterine cervix. I Natl Cancer Inst 1999;91: 252-8.

11 Raffle AE, Alden B, Mackenzie EFD. Detection rates for abnormal cervical smears: what are we screening for? Lancet 1995;345:1469-73. 